Military Technical College

Kobry Elkobbah, Cairo, Egypt

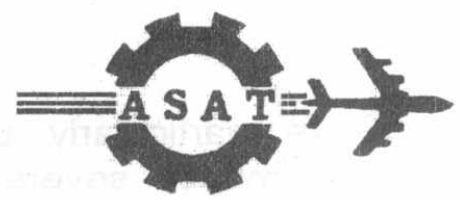

$8^{\text {th }}$ International Conference on Aerospace Sciences \& Aviation Technology

\title{
LINEAR, QUASI-LINEAR AND NONLINEAR MICROSTRIP OSCILLATOR DESIGNS
}

\author{
E.A. Abdallah", M.A. Nassef*, A. Abdel-Nazir"** A.M. El Tager ${ }^{* * * *}$
}

\begin{abstract}
In this paper the analysis and design approaches of microwave oscillators are surveyed and classified into three categories namely, linear, quasi-linear, and nonlinear. An applicable method is chosen from each approach to be implemented using MATLAB package. These MATLAB written programs are used to design an 8.5 $\mathrm{GHz}$ microstrip oscillator. The results of these programs are entered to a powerful CAE package, namely the "Microwave Design System", as initial designs. These designs are enhanced using the available optimization routines. The final optimized linear, quasi-linear, and nonlinear designs are compared using the most powerful nonlinear simulation and the transistor nonlinear model. From these simulations, the performance of each design method can be determined accurately. It is found that the nonlinearly designed oscillator circuit is the most accurate one. This circuit is fabricated and measured to verify the validity of the used simulation techniques. Excellent agreement between theoretical and experimental results is obtained.
\end{abstract}

\section{KEY WORDS}

Microstrip, Oscillators, Linear, Quasi-linear, Nonlinear, Microwave circuits.

\section{NOMENCLATURE}

$l_{1}, l_{2}$

$l_{f}, l_{g}$

$\mathrm{s}_{1}(\mathrm{f})$

$s_{1}(f)$

$s_{2}(f)$

$\mathrm{s}_{3}(\mathrm{t})$

$w, \lambda_{g}$

$\mathrm{Z}_{\mathrm{r}}, \mathrm{Z}_{\mathrm{d}}$ lengths of the single stub matching network lengths of the feedback stub and the gate termination stub the $s_{1}(f)$ after a small change an arbitrary input signal, expressed in frequency domain the frequency response of the linear sub-circuit the response of the nonlinear circuit width of the microstrip line, wavelength inside the microstrip resonator impedance and device impedance

* Prof., Microstrip Dpt., Electronics Research Institute, Cairo, Egypt.

** Associate prof., Dpt. Of Electronic Eng., Military Technical College, Cairo, Egypt.

*** PH.D., R\&D Dpt., Egyptian Armament Authority, Cairo, Egypt.

**** M.Sc. Eng., Dpt. Of Electronic Eng., Military Technical College, Cairo, Egypt. 


\section{INTRODUCTION}

Microstrip solid-state oscillators are particularly useful for space and military applications where weight and size impose severe limitations on the design of the system. Analysis and design approaches of microwave oscillators fall into one of three categories namely, linear, quasi-linear, and nonlinear approaches. The classification of these approaches is shown in Fig. 1.

The linear analysis and design methods are based on small signal S-parameters. Therefore, the prediction of oscillation frequency is our main concern. In this case, it is not possible properly to predict output power or noise performance without scme further oscillator measurements. The closed-loop stability approach allows better control of the stability of the amplifier and the loaded $Q$ of the oscillator, but this method is not easy to use and is inaccurate for the analysis of the highest frequency oscillators. The reflection approach is more convenient for microwave frequencies.

An alternative way combines the advantages of both approaches was obtained [1], to use the gain-loop method (positive feedback) to obtain an approximate output frequency. This output frequency then must be compared with output frequency obtained at different planes of the oscillator using the negative resistance approach. The plane, which achieves the closest frequency to that obtained with the gain-loop method, will be selected to use the negative resistance (reflection) approach.

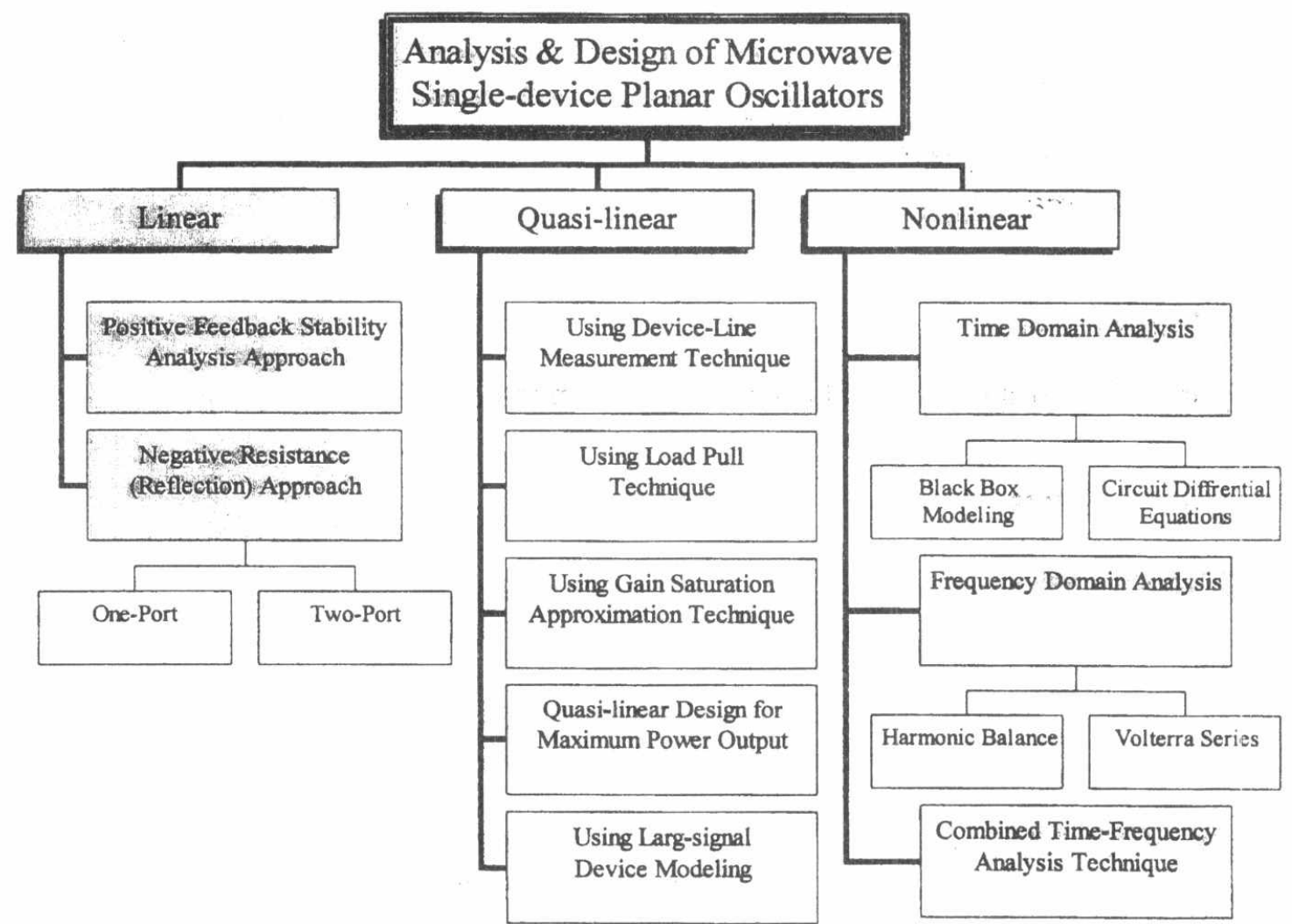

Fig.1. Classification of the analysis and deign methods of the microwave planar single-device oscillators. 
The quasi-linear analysis and design methods are based on the large-signal design techniques such as the device line measurement technique [2], the load pull technique $[3,4]$, and the gain saturation approximation technique $[5,6]$. Some quasilinear approaches are concerned with the maximum power output $[7,8]$, while some others are based on large-signal device modeling techniques[9,10].

The nonlinear analysis and design methods are classified into time-domain, frequency-domain, and combined time-frequency technique [11]. In time-domain technique, the oscillator may be analyzed by solving a set of differential equations as in the conventional circuit theory [12] or by using the black box modeling method [13]. On the other hand, frequency-domain technique may be categorized into harmonic balance [14-16] and Volterra series [17].

The advantages of the time domain nonlinear simulation are:

1. No limitation on signal type (i.e. nonperiodic \& arbitrary signals).

2. Simulation of automatic gain control, and phase locked loop (PLL).

While, the disadvantages are:

1. Can not simulate high-Q resonant or reverberant structures.

2. Can not simulate circuits with long start up times and long delays.

3. Frequency domain simulation (Harmonic Balance) better handle simulation of multiple tone widely spaced frequencies (mixers, converters, etc.).

4. Crystal (XTAL) and surface acoustic (SAW) oscillators are better modeled in Harmonic Balance.

5. This method is time consuming and CPU-intensive.

So, in this paper the harmonic balance technique is used as will be shown in Sec.IV.

\section{LINEAR DESIGN OF A MICROSTRIP OSCILLATOR}

The negative resistance method divides the oscillator into two parts: the negative resistance generator and the resonator [2,18], as shown in Fig.2. The classic oscillator theory of negative resistance establishes that the impedance presented by the resonator must be equal to the impedance presented by the negative resistance generator (with opposite sign) to begin oscillations. The equations

$$
\begin{aligned}
& Z_{r}=-Z_{d} \\
& Y_{r}=-Y_{d}
\end{aligned}
$$

are satisfied in any place in the circuit at the oscillation frequency in the steady -state condition. Although most oscillators operate in large signal conditions, the small signal S parameters are used here. We get the small signal S parameters from data sheets or by measuring the transistor using a network analyzer.

The negative resistance approach has the following Linear design methods:

- The first method is based on maximizing the negative resistance of the active network to obtain a one-port oscillator $[3,4]$. This requires a powerful optimizer to determine appropriate values of the gate termination and the positive feedback.

- The second method is a two-port oscillator [1]. It is easy and straightforward and appropriate for most CAD programs. 
- The third method was proposed by Gonzaleze et. al. [19] especially for crystal oscillators and may be applied for VCOs with minor modifications.

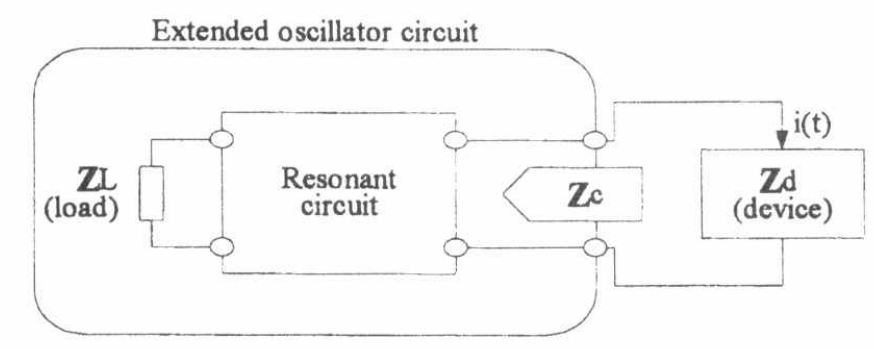

Fig.2. A negative resistance oscillator topology.

A library of programs were written using MATLAB program to facilitate the use of these three oscillator design methods. The oscillator given in Fig. 3 is designed using the implemented program for the second method "CADLinOs.m". The input of this program is the S-parameters of the transistor (NE71083 MESFET) at $V_{d s}=3 \mathrm{~V}$ and $I_{d s}$ $=30 \mathrm{~mA}$. The output design parameters of an $8.5 \mathrm{GHz}$ oscillator are given in Table 1.

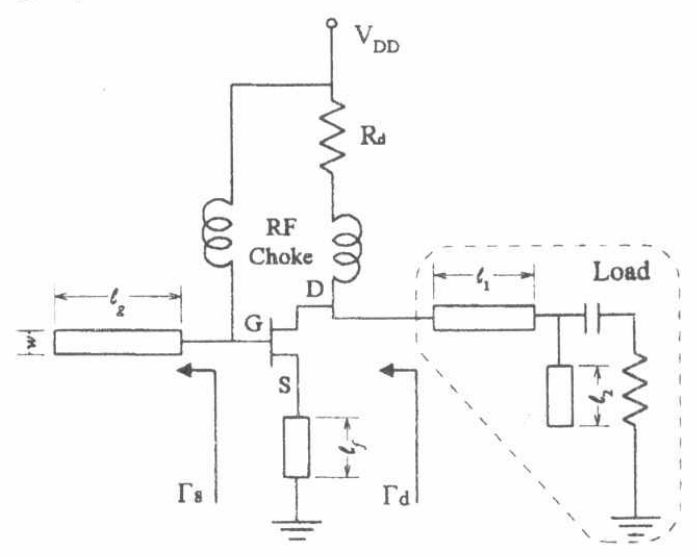

Fig.3. Basic oscillator topology useful in CAD programs.

Table 1. Parameters of the Initial Linear Oscillator.

\begin{tabular}{|l|l|}
\hline parameter & Length in $(\mathbf{m m})$ \\
\hline \hline$\lambda_{g}$ & 25.4 \\
\hline$l_{f}$ & 5.75 \\
\hline$l_{g}$ & 6.25 \\
\hline$l_{l}$ & 2.5 \\
\hline$l_{2}$ & 2 \\
\hline$w$ & 2.4175 \\
\hline
\end{tabular}

\section{QUASI-LINEAR OSCILLATOR DESIGN}

In this paper the gain saturation approximation technique is used as the quasi-linear design method since it is easy to be programmed. This design technique is based on the fact that $S_{21}$ varies more than other $\mathrm{S}$-parameters under large signals. Maeda [6], 
found that small signal measurements are adequate to predict frequency. Johnson [5], suggested that large signal effects produce primarily resistive changes with power much more than the other parameters. It is assumed that all S-parameters except the magnitude of $S_{21}$ are constant under Large-Signal. However, $S_{21}$ is reduced significantly due to transistor saturation. The design using gain saturation approximation procedure is as follow [5]:

- Get the small signal S-parameters (from the manufacturer or by measurements).

- Calculate the amplifier small signal power gain $G$ from the following equation:

$$
G=\frac{\left|S_{21} / S_{12}\right|^{2}-1}{2\left[k\left|S_{21} / S_{12}\right|-1\right]}
$$

Where $k$ is the stability factor of the transistor.

- Calculate the maximum efficient gain $G_{M E}$ associated with the output power from:

$$
G_{M E}=\frac{G-1}{\operatorname{Ln}(G)}
$$

- Substitute $G_{M E}$ for $G$ in Eqn.3 and calculate the reduced value of $\left|\mathrm{S}_{21}\right|$.

- Use the new value of $\left|S_{21}\right|$ to design the embedding circuit of the oscillator.

The gain saturation approximation method is programmed using MATLAB.The written program "Gainsat. $m$ " is applied to the small signal S-parameters of the transistor (NE71083 MESFET), to obtain the large-signal S-parameters approximately. These new parameters are entered to the "CADLinos.m" program to calculate the new structure of the $8.5 \mathrm{GHz}$ oscillator circuit. The parameters given in Table 1 are modified according to the quasi-linear design as shown in Table 2.

Table 2. Parameters of the initial Quasi-linear Oscillator.

\begin{tabular}{|l|l|}
\hline Parameter & Length in $(\mathbf{m m})$ \\
\hline \hline$\lambda_{g}$ & 25.4 \\
\hline$l_{f}$ & 7.524 \\
\hline$l_{g}$ & 5.91 \\
\hline$l_{l}$ & 3.25 \\
\hline$l_{2}$ & 2.9 \\
\hline$w$ & 2.4175 \\
\hline
\end{tabular}

\section{NONLINEAR DESIGN}

The conventional methods which were introduced in Sec.II \& Sec.III and applied using MATLAB have the following sever drawbacks:

a) If the transistor is unconditionally stable at the frequency of interest, a positive feedback is needed. This positive feedback has no simple method to obtain its 
value. In this paper an efficient optimization algorithm will be applied with the following two goals:

$$
\left|S_{11}\right|>1 \quad\left|S_{22}\right|>1
$$

This will maintain a value for the positive feedback network to maximize the reflection coefficient of the active network (transistor \& positives feedback).

b) After load circuit design, a coupling capacitor must be inserted before connecting the $50 \Omega$ load. This capacitor is not included in the calculations of the output circuit, so this may cause a drift in the oscillation conditions. in this paper, the capacitor and its package parasitic are included in the design of the output circuit to confirm the conditions of oscillation.

c) Linear and quasi-linear designs consider the fundamental oscillation frequency only. So, output power, harmonic levels, time-domain waveforms and total harmonic distortion can not be calculated and therefore can not be enhanced. In this work, all these parameters vill be calculated precisely.

The initially designed linear and nonlinear circuits of Sec.II \& Sec.III are enhanced to overcome the drawbacks of a \& b, while the drawback of $c$ is covered through the nonlinear design method of this section. The total oscillator performance of the linear and quasi-linear circuits is optimized using the S-parameters only (i.e. without applying the transistor nonlinear model).

On the other hand, Nonlinear design of oscillator circuits can provide the most accurate prediction of the oscillator performance. But any nonlinear simulation must be applied to a circuit with a transistor nonlinear model.

In this paper an enhancement harmonic balance technique is used as a nonlinear analysis method. As indicrated in Fig.4, the harmonic balance is based on the separation of any circuit int $\%$ two parts:

- First, a sub-circuit 'which contains all the linear elements and the independent voltage and curren'i generators.

- Second, a sub-circuit, which contains all the nonlinear elements.

The linear sub-circuit is analyzed in the frequency domain and the non-linear part in the time domain. The main objective of the harmonic balance method is to find a steady state solution by matching the solutions of these two analyses whatever the studied fre'quency. A Fourier transform is then used to pass from the time domain to the frequ'sency domain. In this case, an error function has to be found and minimized. The prir,ciple of this method is presented in Fig. 4. From a signal $s_{1}(f)$, the response of the linear sub-circuit is obtained $\left[\mathrm{s}_{2}(\mathrm{f})\right]$. After a reverse Fourier transform the signal to the nonlinear circuit is calculated $\left[\mathrm{s}_{2}(\mathrm{t})\right]$. The response of the nonlinear circuit is $s_{3},(t)$. Then $s_{3}(f)$ is obtained by Fourier transform and is compared with the initial $s_{1}(f)$. 'if they are equal, then a steady state is found and $s_{3}(f)$ is the solution. If these two values are not identical, $s_{1}(f)$ must be modified and the overall cycle resumed. 


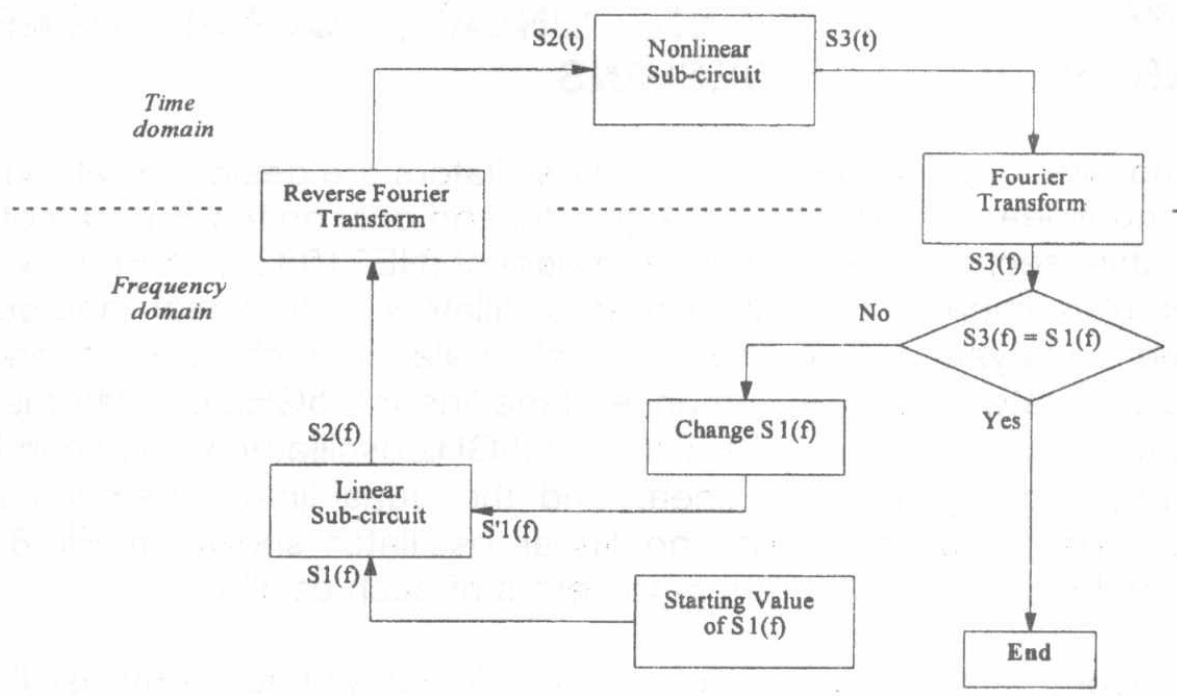

Fig.4. A typical algorithm for the Harmonic Balance nonlinear analysis technique.

To have a high performance oscillator, three points must be established [20]:

1. Only one oscillation frequency is generated.

2. Calculation of the oscillation frequency, the output power and the noise spectrum is Compatible with the specifications.

3. The oscillator frequency is stable as a function of the bias.

The used CAE algorithm for the nonlinear oscillator design is given in Fig. 5. An appropriate nonlinear empirical model is implemented for the used MESFET to prepare the oscillator for nonlinear simulation. The overall microstrip oscillator is designed, simulated and optimized using the nonlinear procedure as shown in Fig.5.

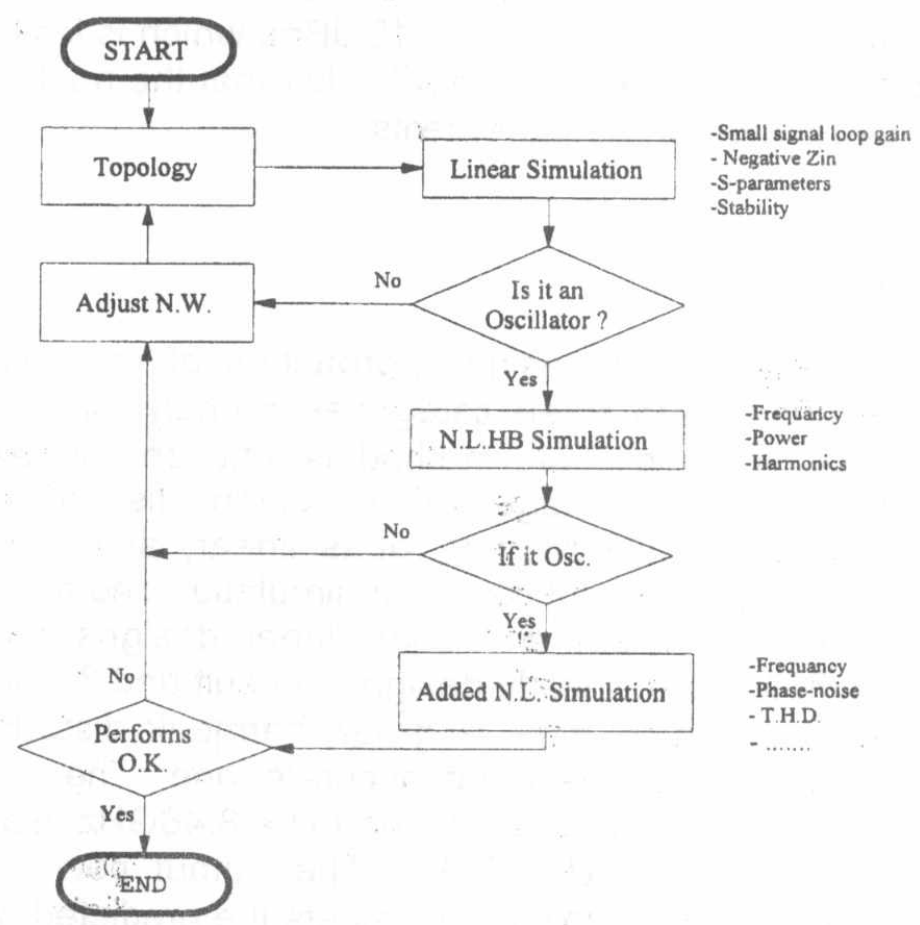

Fig.5. The algorithm of designing an oscillator using the MDS nonlinear simulator. 


\section{COMPARISON BETWEEN LINEAR, QUASI-LINEAR AND NONLINEAR OSCILLATOR DESIGNS}

The linear and the quasi-liriear optimized oscillators are designed without applying the transistor nonlinear model. Their designs depend only on the S-parameters of the transistor. In this section, the nonlinear model of (NE71083 MESFET) will be used with the optimized linear and quasi-linear oscillators. The linear and quasi-linear oscillators are analyzed using the harmonic balance technique to predict their performances accurately. The performance of the linear $8.5 \mathrm{GHz}$ oscillator is shown in Fig.6. The performance of the quasi-linear $8.5 \mathrm{GHz}$ oscillator is shown in Fig.7. It is clear from both figures that the linear and the quasi-linear methods have bad performances compared with the nonlinear oscillator shown in Fig.8. Table 3 compares the values of the important parameters of each oscillator.

Table 3. Comparison between linear, quasi-linear and nonlinear oscillators against the measured Performance.

\begin{tabular}{||l|l|l|l|l||}
\hline & $\begin{array}{l}\text { Linear } \\
\text { Osc. }\end{array}$ & $\begin{array}{l}\text { Quasi- } \\
\text { linear Osc. }\end{array}$ & $\begin{array}{l}\text { Nonlinear } \\
\text { Osc. }\end{array}$ & $\begin{array}{l}\text { Measured } \\
\text { Osc. }\end{array}$ \\
\hline Fundamental frequency $(\mathrm{GHz})$ & 9.167 & 8.66 & 8.5 & 8.464 \\
\hline Output power (in dBm) & 9.2 & 15.9 & 16.78 & 16.16 \\
\hline Output power (ir mW) & 8.3 & 38.9 & 47.64 & 41.3 \\
\hline Total Harmonic Distortion & $70.2 \%$ & $26.1 \%$ & $3.6 \%$ & $3.2 \%$ \\
\hline
\end{tabular}

This nonlinear $8.5 \mathrm{GHz}$ microstrip oscillator circuit is implemented using photolythographic technique. Then it is measured to verify the validity of each design technique. The measured output frequency is $8.464 \mathrm{GHz}$, i.e. shifted from the ideal one by $-36 \mathrm{MHz}$, which represents a good result. So, the frequency accuracy is $0.42 \%$. The measured output power is $16.16 \mathrm{dBm}$, which is less than the simulated one by $0.612 \mathrm{dBm}$. Finally, it is clear from Table1 that the nonlinear oscillator is the closest one to the experimental rneasurements.

\section{CONCLUSION}

In this paper the anzilysis and design approaches of microwave oscillators are surveyed and classified into three categories namely, linear, quasi-linear, and nonlinear approaches. An applicable method is chosen from each approach to be implemented using MATLAB package. All microstrip discontinuities are taken into consideration. The final optimized linear, quasi-linear, and nonlinear designs are compared using the most powerful ronlinear simulation and the transistor norlinear model. It is found that the linear and quasi-linear designs have large frequency deviations $(8.2 \%$ shift for the linearly designed circuit and $3.2 \%$ for the quasi-linear one). These two methods also have a great harmonic distortion. The nonlinearly designizd oscillator circuit is the most accurate one. The measured oscillation frequrancy of the implemented circuit is found to be $8.46 \mathrm{GHz}$. So, it deviates by only $0.4 \%$ from the design target of $8.5 \mathrm{GHz}$. The output power obtained from the irr'plemented nonlinear circuit approximately meets the predicted one. 

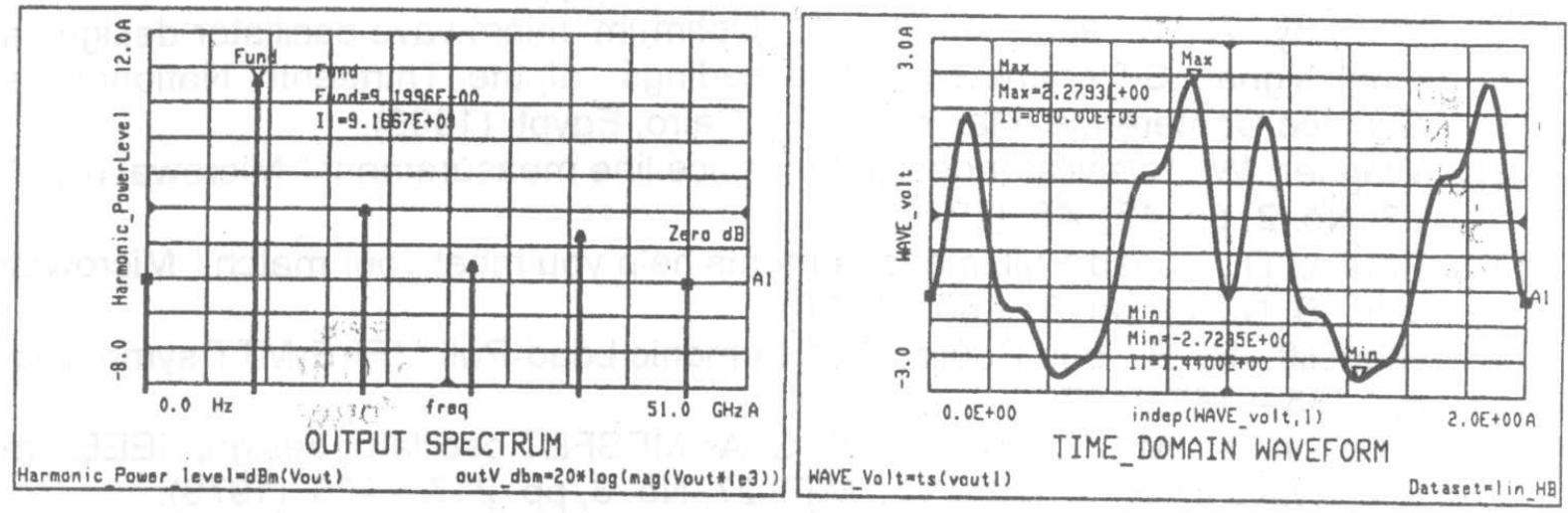

Fig. 6. Harmonic balance simulation output of the optimized $8.5 \mathrm{GHz}$ linear oscillator.
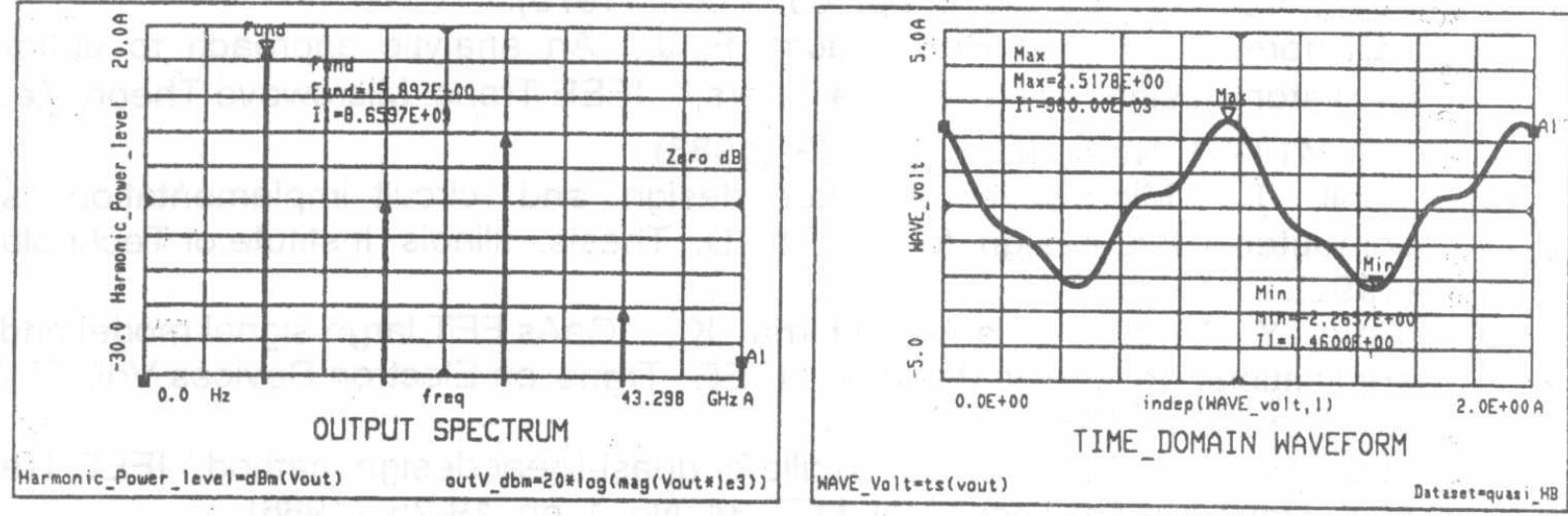

Fig. 7. Harmonic balance simulation output of the optimized quazi-linear oscillator.

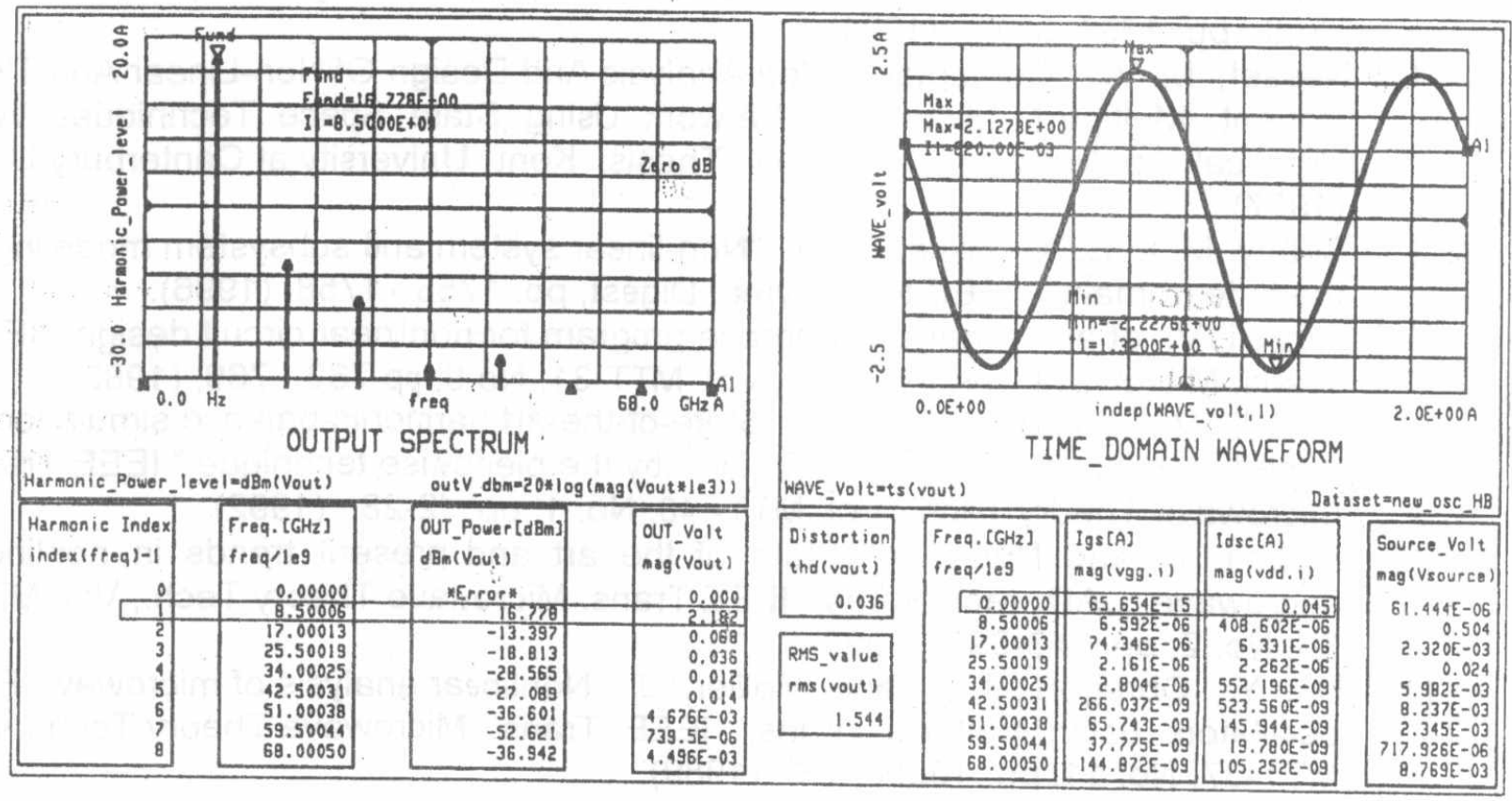

Fig. 8. Harmonic balance simulation output of the optimized nonlinear oscillator. 


\section{REFERENCES,}

[1] Mosf ihy, A. M. and Fouad, M., "Optimum microwave oscillator design using sr.nall-signal S-Parameters," Proceedings of the Thirteenth National Radio Science Conference, March 19 - 21, Cairo, Egypt, (1996).

[L] Wagner, W., "Oscillator design by device line measurement," Microwave J., Vol. 23, No. 2, pp. 43 - 48, (1979).

[3] Poulin, D., "Load-Pull measurements help you meet your match," Microwaves, Vol. 19, No.11, pp. 61 - 65, (1980).

[4] Stancliff, R. B. and Poulin, D., "Harmonic Load-Pull," IEEE-MTT symp. Digest, pp. $185-187,(1979)$.

[5] Johnson, K. M., "Large Signal GaAs MESFET oscillator design," IEEE Trans. Microwave Theory Tech., Vol. MTT-27, No. 3, pp. $217-225$, (1979).

[6] Maeda, M., Kimura, K. and Kodera, H., "Design and performance of X-band oscillators with GaAs Schottky-gate FETs," IEEE Trans. Microwave Theory Tech., Vol. MTT-23, No. 8, pp. 661 - 667, (1975).

[7] Gilmore, R. J. and Rosenbaum, F. J., "An analytic approach to optimum oscillator design using S-Parameters," IEEE Trans. Microwave Theory Tech., Vol. MTT-31, No.8, pp. 633 - 639, (1983).

[8] Nebil, T., "Microwave oscillator design and circuit implementation using computer-aided design tools," $\mathrm{Ph}$. D. Thesis, Illinois Institute of Technology, (1992).

[9] Tajima, Y., Wrora, B. and Mishima, K., "GaAs FET large signal model and its applications to circuit designs," IEEE Trans. on Electron Devices Vol. E:D-28, No. 2 pp. 171 - 175, (1981).

[10] Abe, H., "A GaAs MESFET oscillator quasi-linear design method," IEEE Trans. Microwave Theory Tech., Vol. MTT-34, No. 1, pp. 19-25, (1986).

[11] Schwab, M. H., "Determination of the steady state of an oscillator by a combined time-frequency method," IEEE Trans. Microwave Theory Tech., Vol. MTT-39, No.8, pp. 1391-1402, (1991).

[12] Nassef, M. A. "Computer Aided Analysis And Design Of Non-Linear And Time Variant Multiport Microwave Network Using State Space Techniques With Practical Applications," Ph. D. Thesis, Kent University at Canterbury U. K. (1987).

[13] Sobhy, M. I., E. A. Bakkar, et al. "Non-linear system and subsystem modeling in the time domain.," IEEE MTT Symp. Digest, pp. 1755 - 1758, (1996).

[14] Rizzoli, V., et al. " A general purpose program for nonlinear circuit design," IEEE Trans. Microwave Theory Tech., Vol. MTT-31, No.9, pp.762 - 769, (1983).

[15] Rizzoli, V., Lipparini, A. et. al., "State-of-the-art harmonic-balance simulation of forced nonlinear microwave circuits by the piecenise technique," IEEE Trans. Microwave Theory Tech., Vol. MTT-40, No. 1, pp. 12-28, (1992).

[16] Rizzoli, V. and Neri, A. "State of the art and present trends in nonlinear microwave CAD techniques," IEEE Trans. Microwave Theory Tech., Vol. MTT36, No. 2, pp. 343-365, (1988).

[17] Hu, Y., Obregon, J. J. and Mollier, J., "Nonlinear analysis of microwave FET oscillators using Volterra series," IEEE Trans. Microwave Theory Tech., Vol. MTT-37, No. 11, pp. 1689-1693, (1989). 
[18] Wilson, P. G. and Carver, R. D., "An easy-to-use FET-DRO design procedure suited to most CAD Programs," IEEE Int. Microwave Symp. Digest, pp. 1033 1036, (1989).

[19] Avanic, B., Gonzalez, G. et al., "Negative resistance design for crystal oscillators," INT. J. Electronics, Vol. 67, No. 6, pp.869 - 884, (1989).

[20] Chang, C. R., Steer, M. B. and M. Reese, "Computer-aided analysis of freerunning microwave oscillators," IEEE Trans. Microwave Theory Tech., Vol. MTT-39, No. 10, pp. 1735-1745, (1991). 